\title{
Systemic doxycycline and nonsurgical periodontal treatment in diabetic patients
}

\author{
In type- 1 diabetics, is nonsurgical periodontal treatment with adjunctive \\ doxycycline more effective than nonsurgical periodontal treatment alone?
}

\begin{abstract}
Llambés F, Silvestre F-J, Hernández-Mijares A, Guiha R, Caffesse R. Effect of nonsurgical periodontal treatment with or without doxycycline on the periodontium of type 1 diabetic patients. J Clin Peridontol 2005; 32:915-920
\end{abstract}

Design This was a randomised controlled trial (RCT).

Intervention Group 1 (30 patients) was given oral-hygiene instruction, scaling and root planing, chlorhexidine rinses twice a day and doxycycline ( $100 \mathrm{mg} /$ day for 15 days). Group 2 (30 patients) had the same treatment but without doxycycline.

Outcome measure Plaque index (PI), probing pocket depth (PPD), clinical attachment levels (CAL) and bleeding on probing (BOP) were recorded.

Results Both groups had a measurable improvement in all periodontal parameters, since PI, BOP, PPD and CAL were reduced. The reduction in PPD of $6 \mathrm{~mm}$ and in BOP became statistically significant when doxycycline was used (group 1), however.

Conclusions Although both periodontal treatment regimens are effective in type-1 diabetics, the use of doxycycline as an adjunct provided more significant results when good plaque control was achieved.

\section{Commentary}

This RCT addresses an important issue because, on one hand, diabetes mellitus is an established risk factor for periodontitis and, on the other, chronic infections such as periodontitis impair metabolic control of diabetes mellitus. There are conflicting data regarding the effect of periodontal treatment on metabolic control in people who have diabetes and suffer from periodontitis. Those researchers who have provided periodontal therapy without additional antibiotics failed to observe an effect: studies where antibiotics (tetracycline or its derivatives) were administered in addition have showed metabolic control to be at least temporarily improved.

This study was conducted to assess the clinical effect of systemic doxycycline administered in addition to nonsurgical periodontal treatment (SRP) in type 1 diabetes patients. The trial was wellconducted: assignment to test (SRP plus doxycycline) and control (SRP) was randomised and concealed to examiners and operators. Participants in test and control groups were balanced according to gender, diabetes duration and cigarette consumption, although in the test group there were more patients who had good metabolic control than in the control group. Differences between the groups are described as minimal, but this difference in diabetes control may have an effect on clinical results. Twelve patients who dropped out were replaced by similar new patients to keep the sample size, but reasons for dropout and distribution of dropouts between test and control are not given.

The relevance of this study could also have been significantly improved by using placebo in the control group: ${ }^{1}$ patients were not blinded to treatment assignment. The observed clinical effect of additional doxycycline is rather limited and could be a placebo effect. Further, as well as calculating means of and mean amounts of pockets, a multilevel analysis might have provided stronger evidence for an additional effect of doxycycline while controlling for other influencing factors (eg, diabetic control).

The additional effect of systemic tetracycline on SRP in nondiabetics $^{2,3}$ and type 2 diabetes $^{1}$ is not new and has been shown to persist in the long term. ${ }^{1}$

\section{Practice Point}

In type 1 diabetes patients, doxycycline given adjunctively to SRP has a limited additional clinical benefit.

\section{Peter Eickholz}

Department of Periodontology, Centre for Dental, Oral and Maxillofacial Medicine (Carolinum), Hospital of the Johann Wolfgang Goethe-Universität Frankfurt am Main, Frankfurt/Main, Germany

1. Grossi SG, Skrepcinski FB, DeCaro T, Zambon JJ, Cummins D, Genco RJ. Response to periodontal therapy in diabetics and smokers. J Periodontol 1996; 67:1094-1102.

2. Hung HC, Douglass CW. Meta-analysis of the effect of scaling and root planing surgical treatment and antibiotic therapies on periodontal probing depth and attachment loss. J Clin Periodontol 2002; 29:975-986.

3. Ramberg P, Rosling B, Serino G, Hellström MK, Socransky SS, Lindhe I. The longterm effect of systemic tetracycline used as an adjunct to non-surgical treatment of advanced periodontitis. J Clin Periodontol 2001; 28:446-452.

Evidence-Based Dentistry (2007) 8, 14. doi:10.1038/sj.ebd.6400466 